\title{
V a ria.
}

XII Congrès International de Médecine. Moscou, 7 (19)-14 (26) Août 1897.

Sehr geehrter Herr Redacteur!

Als Vorstand der VIII. Section für Haut- und venerische Krankheiten auf dem XII. Internationalen medicinischen Congress, erlaube ich mir Sie höflichst zu bitten, in Ihrer werthen Zeitschrift die vom Organisations-Comité der VIII. Section aufgestellten Themata mittheilen zu wollen.

\section{THEMATA :}

\section{Dermatologie.}

Actinomycosis. Tuberculosis cutis primitiva. Pathogenie der Area Celsi Acanthosis nigricans. Blennorrhagische Haut-Eruptionen, MalariaEruptionen, Quecksilber-Eruptionen. Behandlung der Sclerodermie. Behandlung des Rhinosclerom.

\section{Venereologie.}

1. Wann hat die Quecksilberbehandlung der Syphilis zu beginnen? Wie lange soll sie dauern? 2. Muss die Behandlung gleich nach dem Ausbruch der allgemeinen Symptome oder vor dem Erscheinen derselben beginnen? 3. Die Veränderungen der Formelemente des Blutes bei $\mathrm{S}_{\mathrm{y}}$ philitikern im condylomatösen Stadium. 4. Die Behandlung der Syphilis mit subcutanen Injectionen löslicher und unlöslicher Präparate.

Abgesehen von den rom Comité der Section ergangenen persönlichen Aufforderungen habe ich die Ehre die hochgeehrten Herren Collegen und Mitglieder des Congresses ergebenst zu bitten entweder dem vom Comité angegebenen Programm beitreten oder ein Thema zum Vortrag: auf dem Congress nach Belieben wählen und das Comité von Ihrem Wunsch unter folgender Adresse in Kenntniss setzen zu wollen:

Moskau, Klinik für Hautkrankheiten, Prof. A. Pospeloff.
Schriftfiihrer:
Vorstand der VIII. Section:
Dr. G. Küs el.
Prof. Dr. A. Pospeloff.

Alle Fragen betreffend wissenschaftliche Angelegenheiten und die: dermatologische Ausstellung mögen an dieselbe Adresse gerichtet werden. 